\title{
FREQUENCY ANALYSIS OF COMPOSITE ANNULAR MEMBRANES
}

\author{
Izabela Zamorska, Stanisław Kukla, Urszula Siedlecka \\ Institute of Mathematics, Czestochowa University of Technology, Poland \\ izabela.zamorska@im.pcz.pl, stanislaw.kukla@im.pcz.pl \\ urszula.siedlecka@im.pcz.pl
}

\begin{abstract}
In this paper, the free vibration of an annular membrane consisting of three concentric segments is considered. The frequency equation and mode shapes are obtained by the use of the Green's function method. A numerical example to vibration problem of nonhomogeneous annular membrane is presented.
\end{abstract}

\section{Introduction}

The problem of transverse vibrations of composite annular membranes has been considered by several authors [1-4]. In the papers $[1,2]$ the free vibration of composite membranes consisting of two annular segments is investigated. The exact solution of the problem and vibration analysis of membranes with discontinuously varying thickness is presented. In reference [2] the finite element method in the analysis was also used. The free vibration problem of annular membrane with many discontinuous variation of the density is the subject of paper [3]. Although the formulation of the problem deals with the vibration of membrane which consist of $m$ segments (each of constant density), the numerical examples concern the antisymmetric modes of composite membrane consisting of two segments. In this case the frequency equation is obtained by setting the determinant of a $4 \times 4$ matrix of coefficients to a derived system of equation, which equals zero. In many papers various methods are applied to solution of the eigenvalue problems. The authors of paper [4] in solving the eigenproblem for annular membrane propose the method of fundamental solution. In this method the free space Green's function are applied.

In this paper the free vibration problem of a composite annular membrane consisting of three segments of constant densities is presented. The solution of the problem (frequency equation, mode shapes) is derived by using the properties of Green's functions corresponding to the Helmholtz operator in an annular domain. An example of numerical frequency analysis is given. 


\section{Formulation and solution of the problem}

The free vibration of a membrane consisting of three annular segments (Fig. 1) is governed by the following differential equations:

$$
\nabla^{2} u_{i}-k_{i}^{2} \frac{\partial^{2} u_{i}}{\partial t^{2}}=-s_{i-1}(t) \frac{\delta\left(r-r_{i-1}\right)}{r}+s_{i}(t) \frac{\delta\left(r-r_{i}\right)}{r}, \quad i=1,2,3
$$

where $u_{i}$ is the displacement of the $i$-th membrane segment, $k_{i}=\sqrt{\rho_{i} / \tilde{s}}, \tilde{s}$ is the tension per unit length, $\rho_{i}$ is the density of $i$-th segment of the membrane, $\delta(\cdot)$ is the Dirac delta function, $\nabla^{2}=\frac{\partial^{2}}{\partial r^{2}}+\frac{1}{r} \frac{\partial}{\partial r}+\frac{1}{r^{2}} \frac{\partial^{2}}{\partial \theta^{2}}$ is the Laplace operator, $r, \theta$ are polar coordinates and $t$ is time. The densities of the membrane change stepwise at circles with radii $r_{1}$ and $r_{2}$. These circles determine the uniform segments of the membrane.

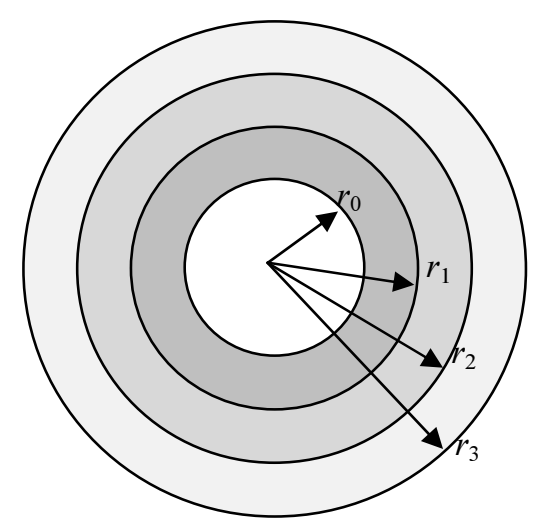

Fig. 1. A sketch of a membrane under study

The functions $u_{i}(i=1,2,3)$ satisfy the continuity conditions:

$$
\begin{gathered}
u_{1}\left(r_{1}, \theta, t\right)=u_{2}\left(r_{1}, \theta, t\right), \quad u_{2}\left(r_{2}, \theta, t\right)=u_{3}\left(r_{2}, \theta, t\right) \\
\left.\frac{\partial u_{1}(r, \theta, t)}{\partial r}\right|_{r=r_{1}}=\left.\frac{\partial u_{2}(r, \theta, t)}{\partial r}\right|_{r=r_{1}},\left.\quad \frac{\partial u_{2}(r, \theta, t)}{\partial r}\right|_{r=r_{2}}=\left.\frac{\partial u_{3}(r, \theta, t)}{\partial r}\right|_{r=r_{2}}
\end{gathered}
$$

and the zero boundary conditions

$$
u_{1}\left(r_{0}, \theta, t\right)=0, \quad u_{3}\left(r_{3}, \theta, t\right)=0
$$

Considering the free vibration of the membrane we assume the functions $u_{i}(r, \theta, t)$ and $s_{i}(t)$ in the form: 


$$
u_{i}(r, \theta, t)=U_{i n}(r) \cos n \theta \cos \omega t, \quad s_{i}(t)=S_{i} \cos \omega t
$$

where $\omega$ is the natural frequency of the membrane. Taken into account equations (4) differential equation (1), continuity and boundary conditions (2)-(3), we obtain:

$$
\begin{gathered}
\mathrm{L}\left[U_{i n}(r)\right]=-S_{i-1} \frac{\delta\left(r-r_{i-1}\right)}{r}+S_{i} \frac{\delta\left(r-r_{i}\right)}{r} \quad i=1,2,3 \\
U_{1 n}\left(r_{1}\right)=U_{2 n}\left(r_{1}\right), \quad U_{2 n}\left(r_{2}\right)=U_{3 n}\left(r_{2}\right) \\
\left.\frac{d U_{1 n}(r)}{d r}\right|_{r=r_{1}}=\left.\frac{d U_{2 n}(r)}{d r}\right|_{r=r_{1}},\left.\frac{d U_{2 n}(r)}{d r}\right|_{r=r_{2}}=\left.\frac{d U_{3 n}(r)}{d r}\right|_{r=r_{2}} \\
U_{1 n}\left(r_{0}\right)=0, \quad U_{3 n}\left(r_{3}\right)=0
\end{gathered}
$$

where $\mathrm{L}_{i n}=\frac{1}{r} \frac{d}{d r}\left(r \frac{d}{d r}\right)+\left(\lambda_{i}^{2}-\frac{n^{2}}{r^{2}}\right)$.

The solution of the problem (5)-(8) can be obtained by the use of the Green's function method. The Green's functions corresponding to the operator $\mathrm{L}_{i n}$ satisfy non-homogeneous differential equation

$$
\mathrm{L}_{i n}\left[G_{i}(r, \zeta)\right]=\frac{\delta(r-\zeta)}{r}, \quad i=1,2,3
$$

and the homogeneous boundary conditions:

$$
\begin{aligned}
& \left.G_{1}(r, \zeta)\right|_{r=r_{0}}=0,\left.\frac{\partial}{\partial r} G_{1}(r, \zeta)\right|_{r=r_{1}}=0 \\
& \left.\frac{\partial}{\partial r} G_{2}(r, \zeta)\right|_{r=r_{1}}=\left.\frac{\partial}{\partial r} G_{2}(r, \zeta)\right|_{r=r_{2}}=0 \\
& \left.\frac{\partial}{\partial r} G_{3}(r, \zeta)\right|_{r=r_{2}}=0,\left.\quad G_{3}(r, \zeta)\right|_{r=r_{3}}=0
\end{aligned}
$$

Multiplying the equation (5) by $r G_{i}(r, \zeta)$ and integrating in interval $\left\langle r_{i-1}, r_{i}\right\rangle$ for $i=1,2,3$, we obtain

$$
\int_{r_{i-1}}^{r_{i}} r \mathrm{~L}_{i n}\left[U_{i n}(\zeta)\right] G_{i}(r, \zeta) d \zeta=\int_{r_{i-1}}^{r_{i}}\left(-S_{i-1} \delta\left(\zeta-r_{i-1}\right)+S_{i} \delta\left(\zeta-r_{i}\right)\right) G_{i}(r, \zeta) d \zeta
$$


Next, we use the self-adjointness of the operator $\mathrm{L}_{i n}$, i.e.:

$$
\int_{a}^{b} r\left[v L_{\text {in }}(u)-u L_{\text {in }}(v)\right] d r=0 \text { for } a \leq r \leq b
$$

for all functions $u, v$ which satisfy the same zero conditions (10) as the functions $G_{i}$. Taking into account (12) in equation (11) we can write

$$
U_{\text {in }}(r)=-S_{i-1} G_{i}\left(r, r_{i-1}\right)+S_{i} G_{i}\left(r, r_{i}\right), \quad r_{i-1} \leq r \leq r_{i}
$$

where we have used the property of Dirac delta function

$$
\int_{a}^{b} f(\zeta) \delta(\zeta-r) d \zeta=f(r) \text { for } a \leq r \leq b
$$

Using (13) in conditions (6) we obtain a system of equations:

$$
\left\{\begin{array}{l}
S_{1 n}\left[G_{1 n}\left(r_{1}, r_{1}\right)+G_{2 n}\left(r_{1}, r_{1}\right)\right]-S_{2 n} G_{2 n}\left(r_{1}, r_{2}\right)=0 \\
-S_{1 n} G_{2 n}\left(r_{2}, r_{1}\right)+S_{2 n}\left[G_{2 n}\left(r_{2}, r_{2}\right)+G_{3 n}\left(r_{2}, r_{2}\right)\right]=0
\end{array}\right.
$$

These equations have a non-trivial solution for the values of parameter $\omega$ which are roots of the frequency equation

$$
\left[G_{1 n}\left(r_{1}, r_{1}\right)+G_{2 n}\left(r_{1}, r_{1}\right)\right]\left[G_{2 n}\left(r_{2}, r_{2}\right)+G_{3 n}\left(r_{2}, r_{2}\right)\right]-G_{2 n}\left(r_{1}, r_{2}\right) G_{2 n}\left(r_{2}, r_{1}\right)=0
$$

Equation (16) is then solved numerically with respect to the non-dimensional frequency $\Omega_{n}=\omega_{n} r_{3} \sqrt{\frac{\rho_{3}}{s}}$.

The mode shapes of vibration corresponding to the determined frequencies we obtain from equation (13)

$$
\begin{array}{ll}
U_{1 n}(r)=S_{1 n} G_{1 n}\left(r, r_{1}\right), & r_{0} \leq r \leq r_{1} \\
U_{2 n}(r)=-S_{1 n} G_{2 n}\left(r, r_{1}\right)+S_{2 n} G_{2 n}\left(r, r_{2}\right), & r_{1}<r \leq r_{2} \\
U_{3 n}(r)=-S_{2 n} G_{3 n}\left(r, r_{2}\right), & r_{2}<r \leq r_{3}
\end{array}
$$

where $S_{2 n}$ is received from (15a)

$$
S_{2 n}=S_{1 n} \frac{G_{1 n}\left(r_{1}, r_{1}\right)+G_{2 n}\left(r_{1}, r_{1}\right)}{G_{2 n}\left(r_{1}, r_{2}\right)}
$$




\section{Numerical examples}

Consider a non-homogeneous annular membrane whose density changes stepwise in a radial direction. The membrane is characterized by radii: $r_{0}=0.2$, $r_{1}=0.4, r_{2}=0.6, r_{3}=0.8$, and by ratios of densities of the segments: $\sigma_{1}=\frac{\rho_{1}}{\rho_{3}}$ and $\sigma_{2}=\frac{\rho_{2}}{\rho_{3}}$. The eigenfrequencies of the membrane we calculate numerically by using the frequency equation (16). The bisection method was applied. The nondimensional frequencies corresponding to symmetric modes of vibrations for various values of ratios $\sigma_{1}$ and $\sigma_{2}$ are presented in Table 1 . The comparison of the results presented in the table leads to the conclusion that increase of the densities ratios causes decrease of the frequencies of the membrane.

Table 1

First five frequency values $\Omega_{0 i}=\omega_{0 i} r_{3} \sqrt{\frac{\rho_{3}}{s}}$, for nonhomogeneous membrane shown in Figure 1 for various values of $\sigma_{1}$ and $\sigma_{2}$

\begin{tabular}{|c|c|c|c|c|c|}
\hline \multirow{2}{*}{$\sigma_{1}$} & $\Omega_{01}$ & $\Omega_{02}$ & $\Omega_{03}$ & $\Omega_{04}$ & $\Omega_{05}$ \\
\cline { 2 - 6 } & \multicolumn{5}{|c|}{$\sigma_{2}=0.1$} \\
\hline 0.1 & 7.22575 & 19.09385 & 31.38584 & 43.66073 & 55.38157 \\
\hline 1.0 & 6.12412 & 9.09007 & 18.70560 & 20.28878 & 32.31386 \\
\hline 5.0 & 1.43392 & 3.82080 & 6.26935 & 7.97735 & 8.94993 \\
\hline 10.0 & 0.71866 & 1.91728 & 3.15893 & 4.40742 & 5.65656 \\
\hline \multicolumn{5}{|c|}{$\sigma_{2}=1.0$} \\
\hline 0.1 & 4.48688 & 10.11479 & 16.13784 & 22.28729 & 28.48738 \\
\hline 1.0 & 4.09769 & 8.32377 & 12.52867 & 16.72628 & 20.92059 \\
\hline 5.0 & 1.40561 & 3.70511 & 5.74119 & 6.78442 & 8.83797 \\
\hline 10.0 & 0.71516 & 1.90563 & 3.13651 & 4.36547 & 5.54419 \\
\hline \multicolumn{5}{|c|}{$\sigma_{2}=5.0$} \\
\hline 0.1 & 1.01913 & 2.89382 & 5.18590 & 7.56466 & 9.91070 \\
\hline 1.0 & 1.01416 & 2.86149 & 5.11402 & 7.43692 & 9.68276 \\
\hline 5.0 & 0.89200 & 2.01103 & 3.20914 & 4.43146 & 5.66214 \\
\hline 10.0 & 0.62994 & 1.43690 & 2.08907 & 3.04102 & 3.82215 \\
\hline \multicolumn{5}{|c|}{$\sigma_{2}=10.0$} \\
\hline 0.1 & 0.51144 & 1.45959 & 2.62192 & 3.83689 & 5.06853 \\
\hline 1.0 & 0.51081 & 1.45556 & 2.61350 & 3.82366 & 5.04984 \\
\hline 5.0 & 0.49536 & 1.33866 & 2.20651 & 2.87151 & 3.78985 \\
\hline 10.0 & 0.44705 & 1.00976 & 1.61248 & 2.22774 & 2.84796 \\
\hline
\end{tabular}




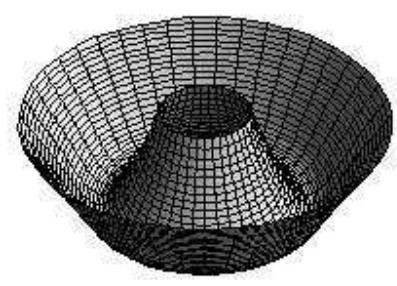

(a)

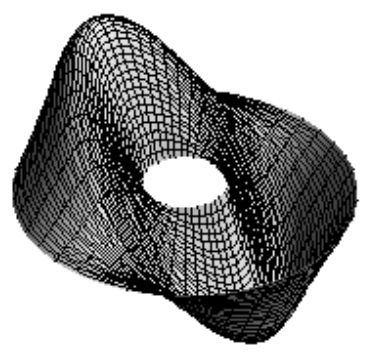

(c)

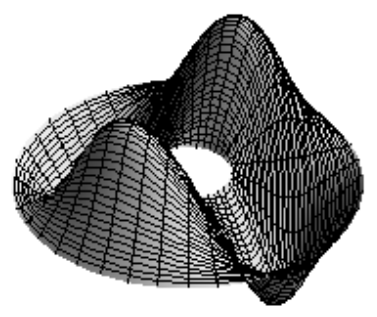

(e)

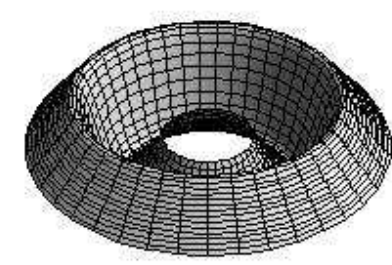

(b)

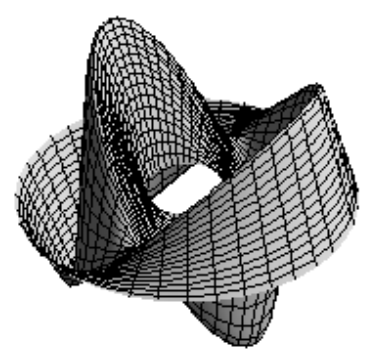

(d)

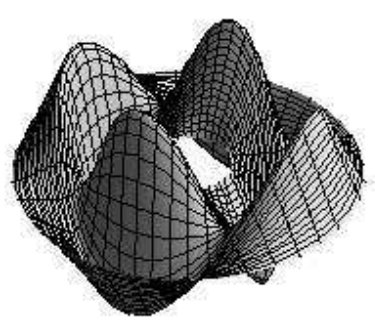

(f)

Fig. 2. Mode shapes for an annular membrane: a) $\Omega_{01}=1.01416$, b) $\Omega_{02}=2.86149$,

c) $\Omega_{11}=1.09474$, d) $\Omega_{12}=2.90734$, e) $\Omega_{21}=1.29468$, f) $\Omega_{22}=3.03193$

Mode shapes of the annular membrane shown in Figure 1 for $r_{0}=0.2, r_{1}=0.4$, $r_{2}=0.6, r_{3}=0.8$, and $\sigma_{1}=1.0 ; \sigma_{2}=5.0$ are presented in Figure 2. The surfaces are plotted on the basis of formulas (17) and (18). The first two surfaces (Fig. 2a, b) obtained for $n=0$, represent the axisymmetric modes of vibration and the remained for $n=1$ and $n=2$, present the antisymmetric modes of vibrations.

\section{Conclusions}

In this paper the solution to the problem of free vibration of an annular membrane consisting of three concentric segments, each of constant density is present- 
ed. The frequency equation and mode shapes are expressed by Green's functions corresponding to relevant Helmholtz problems. Numerical results presented in the table show the effect of change the densities of the membrane segments on the eigenfrequencies of the system: increase of the ratios of densities causes a decrease of the free vibration frequencies. The presented here the method to solution of the free vibration problem of a membrane consisting of the three segments can be generalized on the annular membrane consisting of an arbitrary number of concentric segments.

\section{References}

[1] Laura P.A.A., Rossit C.A., La Malfa S., Transverse vibrations of composite, circular annular membranes: exact solution, Journal of Sound and Vibration 1998, 216(1), 190-193.

[2] Rossit C.A., La Malfa S., Laura P.A.A., Antisymmetric modes of vibrations of composite, doubly-connected membranes, Journal of Sound and Vibration 1998, 217(1), 191-195.

[3] Marciniec A., Noga S., Natural frequencies and mode shapes of a composite annular membrane, Vibrations in Physical Systems 2010, 24, 277-282.

[4] Lee Y.-T., Chen I-L., Chen J.-T., Eigensolution of annular membrane using the method of fundamental solutions, The $27^{\text {th }}$ Conference on Theoretical and Applied Mechanics, Taiwan, R.O.C., 12-13 December 2003.

[5] Kukla S., Siedlecka U., Zamorska I., Green's functions for interior and exterior Helmholtz problems, Scientific Research of the Institute of Mathematics and Computer Science Czestochowa University of Technology 2012, 1(11), 53-62.

[6] Wolfram S., The Mathematica Book, Wolfram Media, 5th ed., 2003. 